\title{
Presentación del monográfico "La prostitución: entre viejos privilegios masculinos y nuevos imaginarios neoliberales"
}

\author{
Presentation of the special issue \\ "Prostitution: between old male privileges and new \\ neoliberal imaginaries"
}

Beatriz Ranea Triviño

Recibido: 30/06/2018

Aceptado: 02/09/2018

El viejo canon de la prostitución ha desaparecido y ha sido sustituido por otro nuevo que adopta la forma de una gran industria interconectada, con formas de funcionamiento propias de una gran corporación del capitalismo global y con un pie en la economía lícita y otro en la ilícita.

Rosa Cobo

El estudio de la prostitución ha de inscribirse dentro del contexto social en el que se produce y que posibilita su existencia de tal forma que podemos definirla como una institución masculina patriarcal -como establecía Victoria Sau (2000) en su Diccionario ideológico feminista- en la que el capitalismo neoliberal ha encontrado un negocio muy lucrativo. A día de hoy, el significado sociopolítico de la prostitución mantiene su origen patriarcal atravesado por nuevas lógicas propias de la era del capitalismo neoliberal. Por ello, en este monográfico el análisis de la prostitución aparece imbricado en la encrucijada entre capitalismo y patriarcado. En los artículos se analiza y reflexiona sobre diferentes elementos que configuran la prostitución en la sociedad contemporánea: la conversión en una macroindustria transnacional que obtiene grandes beneficios a costa de la explotación sexual de mujeres y niñas; las nuevas definiciones neoliberales que transforman los imaginarios legitimadores

Beatriz Ranea Triviño es investigadora doctoral de la Universidad Complutense de Madrid (Madrid, España). Correo electrónico: $\underline{\text { b.ranea@ucm.es. ID https:/ / orcid.org/0000-0002-4595-4436 }}$

Cómo citar esta presentación: Ranea, Beatriz (2018). Presentación del monográfico. La prostitución: entre viejos privilegios masculinos y nuevos imaginarios neoliberales. Atlánticas. Revista Internacional de Estudios Feministas, 3 (1), 1-12. doi: http:/ / dx.doi.org/10.17979/arief.2018.3.1.3540 
de la misma; o como la prostitución gana importancia como un escenario de representación de un modelo determinado de masculinidad.

En cuanto a los "viejos" privilegios masculinos, la perspectiva de género y feminista nos permite comprender por qué las mujeres conforman mayoritariamente la "oferta" de prostitución ${ }^{1}$ y por qué los hombres son quienes devienen de forma mayoritaria demanda. La prostitución es una institución que garantiza que haya hombres que de forma individual o en grupo accedan al cuerpo de mujeres que no les desean, un hecho que fuera de la prostitución se consigue mediante violencia explícita o intimidación. Es decir, las políticas del deseo en las sociedades patriarcales posibilitan que el deseo masculino se imponga mediante una transacción económica que forma parte del continuum de devaluación de las mujeres y de negación e invisibilización de su subjetividad (sexual). En las sociedades patriarcales, la masculinidad hegemónica se construye, en gran medida, sobre una serie de privilegios (imaginados como derechos) sobre las mujeres y resto de sujetos no hegemónicos. Garantizarse el acceso a los cuerpos de las mujeres, aunque éstas no les deseen, es un viejo privilegio masculino.

En este sentido, hay que destacar que las mujeres son representadas como mero cuerpo dentro del orden social patriarcal (Posada Kubissa, 2015), y la prostitución es una de las instituciones sociales dónde se hace más evidente esa asimilación de las mujeres-cuerpo. Las mujeres son representadas como cuerpos sin individualidad, ni subjetividad reconocida y, por tanto, forman parte de las "idénticas" (Amorós 1987), una condición necesaria para que puedan ser percibidas como bienes intercambiables en el mercado de la prostitución.

Además, representar a las mujeres como meros cuerpos sin subjetividad posibilita asimismo concebir su sexualidad como un elemento negado y ajeno, y de esta manera, un producto mercantilizable. La sexualidad de las mujeres se desvincula de su subjetividad y de su corporeidad y permite conceptualizarla

\footnotetext{
${ }^{1}$ Sin obviar que también hay mujeres transexuales y hombres situación de prostitución. Según Médicos del Mundo, en su memoria de 2016 recogía los siguientes datos de las personas atendidas en contextos de prostitución: el $91,31 \%$ fueron mujeres, el 5,13\% transexuales y el 3,56\% hombres. Así, si analizamos el género de la "oferta" de prostitución hay que destacar los tres grupos sociales que se encuentran en prostitución son: mujeres, mujeres transexuales y hombres que mantienen sexo con hombres.

Si algo tienen en común los tres grupos que se encuentran en prostitución es, por un lado, que la demanda es mayoritariamente masculina en los tres casos; y por otro lado, la discriminación y devaluación social que sufren los tres grupos sociales en prostitución. Los procesos de mercantilización del cuerpo y la sexualidad son posibles porque son personas que se encuentra en un lugar menos ventajoso en la estructura social. En sociedades con fuertes desigualdades socioeconómicas, las corporeidades devaluadas son representados socialmente como mercantilizables. Sin embargo, estas tres tipologías de prostitución no son directamente comparables porque cada una tiene sus especificidades, en este monográfico analizamos fundamentalmente la prostitución de mujeres.
} 
como un objeto o un "servicio". No obstante, no ocurre lo mismo con la representación de la sexualidad en el caso de los hombres: para muchos de los hombres que demandan prostitución, la sexualidad aparece pensada, imaginada y ejecutada como un elemento imposible de ser desvinculado de su propio cuerpo y su propia subjetividad. En el imaginario sociosexual patriarcal, la sexualidad masculina se representa como una "necesidad" fisiológica, esto es, como una "necesidad" corporal. Este imaginario que esencializa la masculinidad ha sido uno de los grandes legitimadores de las violencias sexuales porque ficciona la sexualidad masculinidad como una "necesidad" llegando a ubicarla incluso en el terreno de los instintos que se han de satisfacerse de una manera $u$ otra.

Por otro lado, podemos afirmar que la percepción de la sexualidad como mercantilizable convertida en "servicios sexuales" plantea un problema conceptual que ha de ser abordado. En la actualidad, la sexualidad se reconoce como uno de los pilares del proceso de subjetivación de las personas y la orientación sexual se ha convertido en uno de los ejes fundamentales sobre los que se cimientan las identidades contemporáneas. No obstante, a pesar de la relevancia de la sexualidad, cuando se aborda la cuestión de la prostitución, la sexualidad aparece significada de una forma completamente distinta: se disocia de los procesos de subjetivación y del cuerpo de las personas en prostitución y, de esta manera, es representada como un servicio o como un producto. Si la sexualidad es disociable del cuerpo, y no se le concede la relevancia social que tiene, carecería de sentido conceptualizar e identificar -como lo hemos hecho- el elemento sexual de la violencia contra las mujeres. Desde hace unos años, estamos viviendo un momento histórico de movilizaciones feministas que, entre otros temas, han situado la violencia sexual en el centro de la agenda política. En este contexto es imprescindible reflexionar sobre la construcción de la sexualidad masculina que produce y legitima la violencia sexual y que representa el acceso a los cuerpos de las mujeres como un "derecho" ya sea mediante violencia explícita, intimidación y/o mediante el pago. La masculinidad hegemónica supone una barrera hacia el reconocimiento de la subjetividad (sexual) de las mujeres. Dentro de este modelo de masculinidad, la sexualidad es expresada como deseo masculino sin dar cuenta de la reciprocidad que implicaría reconocer el deseo de las mujeres.

Por añadidura, la prostitución es una vieja institución que desde hace unas 
décadas gana relevancia en el mantenimiento del orden patriarcal porque puede ser interpretada como un espacio de reconstrucción subjetiva masculina ante la quiebra de algunos de los pilares sobre los que se sostenía tradicionalmente la masculinidad. Por un lado, asistimos a una fuerte interpelación feminista a las formas patriarcales de la masculinidad que, a su vez, reaccionan para mantener el status quo patriarcal; y de otro lado, han quebrado algunas de las instituciones que garantizaban la reafirmación de la masculinidad como el trabajo estable o la familia patriarcal (Gil Calvo, 2006). Es decir, los pilares sobre los que se cimentaba la masculinidad se desestabilizan, y por contrapartida, se resignifican viejas instituciones como la prostitución. Como sostienen Rosa Cobo (2017) y Beatriz Gimeno (2018) -en este mismo monográfico-, los hombres encuentran en la prostitución escenarios de reconstrucción subjetiva, tanto de forma individual como colectiva. Los contextos de prostitución adquieren mayor importancia como refugios de una masculinidad que fuera de estos espacios es difícil representar. Esto es, para muchos hombres que demandan prostitución, los espacios prostibularios son aquellos "donde los hombres son todavía hombres y las mujeres son todavía mujeres" (Marttila, 2008:41), donde se restituye el orden natural de las cosas, las relaciones de género en términos patriarcales.

En contra de todo lo expuesto, el marco interpretativo de la prostitución como "trabajo sexual" tiende a invisibilizar el análisis crítico de la demanda de prostitución y, por tanto, no conecta la prostitución con la construcción de la sexualidad masculina. Se puede afirmar que este marco no transgrede totalmente el pensamiento androcéntrico, donde se evita aludir a la responsabilidad de los hombres porque la masculinidad no es pensada como problemática o como una situación privilegiada. Como sostiene Michael Kimmel (2018) la invisibilidad es una cuestión política, porque aquello que constituye la norma y los privilegios que acompañan se invisibilizan. Por esto, los hombres tradicionalmente no han sido estudiados como sujetos genéricos sino que se ha señalado lo abyecto, todo aquello que no constituye lo normativo: las mujeres, las personas pobres, las racializadas, las homosexuales... El privilegio masculino se ubica en el no hacerse cargo de uno mismo y por esto, se señala, se responsabiliza y se estigmatiza a las otras.

En cuanto a los nuevos mercados e imaginarios neoliberales, la prostitución se ha convertido en una macroindustria que moviliza ingentes cantidades de 
dinero a escala global. Teóricas contemporáneas como Rosa Cobo (2017) señalan que hoy día no es posible analizar el crecimiento de la prostitución y de la trata sin situarla en el centro de la barbarie capitalista. Solo así es posible acercarse al significado sociopolítico que adquiere la prostitución no sólo como resorte patriarcal sino también como mercado altamente lucrativo a costa de la explotación sexual de las mujeres y niñas.

En aras de complejizar el análisis de la prostitución dentro del contexto capitalista, se ha de reflexionar sobre las desigualdades sociales que generan expulsiones de mujeres al mercado prostitucional (Cobo, 2017). Las realidades de los contextos de prostitución han de ser analizadas desde una perspectiva interseccional que dé cuenta de los distintos ejes de vulnerabilidad que lo atraviesan. En los espacios de prostitución nos encontramos con mujeres tanto autóctonas como, sobre todo, migrantes que suelen tener en común las condiciones de precariedad económica que, además, pueden estar atravesadas por discriminaciones y vulnerabilidad por el estatus migratorio; la etnicidad; la ausencia de redes de apoyo familiar, comunitario, social o institucional; la edad; la diversidad funcional; los consumos problemáticos de drogas; o antecedentes de violencia de género.

En un contexto en el que las políticas neoliberales han incrementado las desigualdades sociales y en el que enfrentamos devastadoras cifras de pobreza, es una irresponsabilidad -o un ejercicio de cinismo como señala Nuria Varela (2017)- obviar la pobreza, la privación material, la precariedad y la clase social en el análisis de esta realidad. Son diversos los estudios que señalan la relación entre la feminización de la pobreza y la prostitución (Cobo, 2017; Bredy et al., 2014; Castellanos y Ranea Triviño, 2013, 2014; García Cuesta et al., 2011; Fernández Viguera y Munárriz Gómara, 2008; entre otros) y si nos detenemos en las cifras del último informe AROPE sobre la situación de la pobreza (EAPN, 2018) observamos que en el Estado español un 27,9\% de la población se encuentra en riesgo de pobreza. En cuando a la tasa de carencia material severa se destaca que un $6,2 \%$ de las mujeres se enfrenten a esta situación.

Por ello, es importante destacar que el mercado de la prostitución necesita de la pobreza -y la feminización de la misma- para "nutrirse", esto es, necesita que las desigualdades sociales se sigan reproduciendo. Un Estado que normaliza la prostitución como "trabajo sexual" deja de actuar en favor de la igualdad de oportunidades de las mujeres en situación de mayor desventaja social porque 
para éstas aparecerá la prostitución como una alternativa (Gimeno, 2012). En ese escenario, carecerían de sentido las políticas que promuevan la igualdad de oportunidades para las mujeres, o las políticas centradas en la redistribución de la riqueza, o las políticas de protección social porque las expulsiones de mujeres crean nichos de precariedad donde la prostitución aparece como economía de supervivencia. En este sentido, existe una relación directa entre el bienestar de una comunidad y el número de mujeres autóctonas que se prostituyen en la misma (Cobo, 2007). Es decir, cuando aumenta el bienestar de una sociedad y se toman medidas en pro de la igualdad de oportunidades, disminuye el número de mujeres autóctonas vinculadas a la prostitución y son mujeres de otros contextos más empobrecidos quienes llenan los espacios de prostitución. Esto ocurre a lo largo y ancho de los países Occidentales y, de esta manera, la industria de la prostitución (re)produce lógicas coloniales. Sheila Jeffreys (2009) se refiere al colonialismo sexual para dar cuenta de este fenómeno: países Occidentales que nutren sus prostíbulos (y resto de espacios de prostitución) mediante la colonización de cuerpos de mujeres de otras regiones más empobrecidas o de minorías culturas. Esta colonización se produce en dos sentidos y en dos procesos que se han dado de forma simultánea en estos años de desarrollo de la industria de la prostitución: de un lado, la industria coloniza cuerpos de mujeres en países Occidentales; de otro lado, a través del fenómeno conocido como el "turismo sexual", la industria posibilita a los hombres occidentales viajar a otros países para pagar por sexo comercial. Es importante destacar que este colonialismo sexual genera un racismo sexualizado que es innegable por la forma en la que la demanda de prostitución estereotipa a las mujeres siguiendo construcciones culturales etnocéntricas (Ranea, 2016).

Además, es necesario destacar que el mercado prostitucional se sirve de las políticas fuertemente restrictivas a la inmigración porque la situación administrativa irregular aumenta la vulnerabilidad de las mujeres que carecen de derechos básicos y por tanto, no pueden acceder ni al mercado de trabajo ni a las medidas de protección social. En estas circunstancias, para algunas mujeres en situación administrativa irregular, aparece la prostitución como una de las pocas opciones de conseguir ingresos dentro de los circuitos de economía informal. En la investigación llevada a cabo por Izcara-Palacios y AndradeRubio (2016) sobre la situación de las mujeres centroamericanas víctimas de trata con fines de explotación sexual en Estados Unidos, señalan que para la industria del sexo no sólo es importante la captación de mujeres sino también 
los "mecanismos de expulsión" de aquellas mujeres que no resultan "rentables" en el mercado de la prostitución. En el caso que analizan, el sistema prostitucional se vería beneficiado por las políticas restrictivas a la inmigración estadounidenses, ya que estas mujeres "no rentables" son expuestas para ser deportadas de nuevo a sus países de origen. Así, el sistema prostitucional se beneficia de las políticas restrictivas a la inmigración tanto para captar mujeres como para desecharlas.

Por todo esto, se puede afirmar que el sistema prostitucional necesita los procesos de feminización de la pobreza y precarización de la existencia para seguir generando "oferta"; mientras, por otro lado, invertirá esfuerzos en incrementar la demanda masculina.

En este escenario, el cuerpo de las mujeres constituye un campo de batalla significado en el marco neoliberal como un cuerpo-territorio o, incluso, un cuerpo-máquina del que es posible obtener grandes beneficios. Se representa el cuerpo de las mujeres de forma parecida a las representaciones que se realizan en el orden capitalista de la naturaleza donde ésta aparece como un instrumento para obtener recursos y materias primas sin prestar atención a las consecuencias que supone la explotación de la misma. Como señala Rosa Cobo (2018) se sigue "en la prostitución de mujeres la lógica extractivista, característica de la fase de capitalismo que vivimos actualmente. Es decir, la lógica de sacar beneficios a toda costa".

Además, se ha de reflexionar sobre el proceso de colonización del imaginario colectivo por parte del marco neoliberal que ha supuesto una transformación de los discursos legitimadores de esta institución patriarcal. Asistimos a la construcción de marcos de interpretación, definiciones y simplificaciones de la realidad de la prostitución propia del neoliberalismo que despolitiza las desigualdades sociales y presenta todo intercambio económico al margen de la estructura social y las relaciones de poder. Así, la relación de prostitución queda en manos de un mercado supuestamente neutro en el que "cliente" y prostituta aparecen en el mismo plano: uno tiene dinero y otra acepta. Se redefinen los argumentos legitimadores de la prostitución en base a decisiones individuales de cada uno de los sujetos ocultando las desigualdades sociales que posibilitan la prostitución. 
Por todo lo expuesto, en este monográfico se plantea la necesidad de reflexionar críticamente sobre los diferentes elementos que atraviesan la prostitución como: la industria de la prostitución y su crecimiento exponencial a escala global; las conexiones entre la industria de la prostitución y la trata de mujeres y niñas con fines de explotación sexual; los procesos de expropiación de los cuerpos de las mujeres en esta fase del capitalismo tardío; el rol fundamental de los hombres que demandan prostitución y el modelo de masculinidad hegemónica que perpetúan a través del consumo de prostitución; la responsabilidad de los Estados que legitiman la prostitución; y el papel de la sociedad y las narrativas que contribuyen a normalizar la prostitución.

Así, diferentes autoras reflexionan críticamente desde distintas disciplinas sobre el papel de la prostitución en el mantenimiento y reconstrucción de un modelo de masculinidad que se muestra como un resorte del orden de género patriarcal; y a su vez, nos plantean un análisis de gran interés sobre la construcción de nuevos imaginarios imbricados en el contexto neoliberal y que contribuyen a la legitimación social de la prostitución:

Beatriz Gimeno analiza las transformaciones que han dado lugar a la megaindustria transnacional de la prostitución cuyo crecimiento ha ido de la mano de su resignificación en el contexto neoliberal. El neoliberalismo ha posicionado a muchos hombres en una situación en la que con anterioridad solo se encontraban las mujeres y por ello, la prostitución como institución reguladora del orden de género gana importancia como escenario de reconstrucción subjetiva para los hombres. Esto es, los contextos de prostitución se han convertido en refugios necesarios para reconstruir la masculinidad hegemónica.

Juana Gallego presenta un análisis acerca de la importancia de los medios de comunicación y de las ficciones fílmicas para visibilizar o invisibilizar prácticas sociales, así como para cambiar "marcos discursivos" y el sistema de valores en torno a dichas prácticas sociales. En lo referente al relato fílmico, destaca cómo la prostituta es un personaje femenino recurrente que se ha representado de diversas formas en cuyos extremos se encuentra la esclava víctima de trata y, por otro lado, la actual trabajadora sexual que tiende a ser presentada "de manera mucho más glamurosa, apetecible, sencilla, sin apenas inconvenientes, fácil y sobre todo muy, muy lucrativa, lo cual es una propuesta legitimadora". 
Silvia Pérez Freire presenta los resultados obtenidos de la investigación que ha realizado sobre imaginarios sociales de la prostitución y la trata sexual. La investigadora explica los mecanismos de supervivencia que establecen las mujeres víctimas de trata; mientras que en el caso de los hombres que demandan prostitución se establecen mecanismos de apropiación de los cuerpos de las mujeres, y una cosmovisión legitimadora de sus acciones. Por otro lado, Pérez Freire constata como la legitimación social de la prostitución provoca la invisibilización de la explotación sexual y la trata con fines de explotación sexual.

Carmen Delgado parte de las evidencias empíricas disponibles para cuestionar el modelo regulacionista de la prostitución. La autora discute la validez no sólo científica sino teórica de un modelo que se basa en excepciones que representan la prostitución como empoderadora y liberadora. Además, siguiendo a Nancy Fraser, Delgado señala cómo ha permeado el marco neoliberal en estos planteamientos feministas que se refieren a acuerdos mutuos entre dos partes sin inscribir las relaciones en el orden desigual de género.

Peter Szil señala la responsabilidad de los hombres en el sostenimiento de la prostitución y la trata de mujeres con fines de explotación sexual. En el proceso de creación de la demanda de prostitución en las sociedades contemporáneas, Szil, reflexiona sobre el papel que tiene la pornografía hegemónica. Es decir, señala la conexión entre la construcción del imaginario sociosexual masculino atravesado por la pornografía hegemónica y el consumo de prostitución.

\section{Esther Torrado Martín-Palomino, Yasmina Romero Morales y Josué Gutiérrez}

Barroso presentan una parte de los principales resultados de la investigación Estudio sobre la prostitución de mujeres en Canarias. En esta investigación han realizado entrevistas en profundidad a mujeres prostituidas y Focus Group con profesionales que intervenían directa o indirectamente con estas mujeres. Por otro lado, se han llevado a cabo entrevistas en profundidad a hombres consumidores de prostitución. Así como una encuesta telefónica a la población de las siete islas para conocer las percepciones sobre la prostitución de mujeres. Se presentan en este informe los resultados de dicha encuesta.

El monográfico cierra con dos entrevistas a dos figuras de una relevancia 
fundamental tanto del feminismo de los años 70-80 como en la actualidad:

Amelia Tiganus, superviviente de trata y activista, una de las figuras más representativas del pensamiento abolicionista a día de hoy. A lo largo de la entrevista Tiganus reflexiona sobre diversas cuestiones como: el proceso de fabricación de la prostituta; la responsabilidad de los hombres que demandan prostitución; el papel del Estado como Estado proxeneta que legitima y permite la prostitución; la importancia de la pornografía hegemónica como reproductora de un imaginario sociosexual fuertemente patriarcal; y por último, sobre el feminismo y el proceso vivido de víctima a superviviente y de superviviente a activista.

Kathleen Barry como teórica del feminismo radical a lo largo de la entrevista señala la importancia de conectar la prostitución con las diversas formas que adopta la violencia sexual contra las mujeres. Reflexiona críticamente sobre la masculinidad y los procesos de socialización masculina ligados a la violencia y la ausencia de empatía, destacando la vinculación entre masculinidad y militarismo. Además, Barry proporciona un inspirador discurso sobre la relevancia de la sororidad y los consciousness raising groups.

\section{BIBLIOGRAFÍA}

Amorós, Celia (1987). Espacio de los iguales, espacio de las idénticas. Notas sobre poder y principio de individuación. Arbor: Ciencia, pensamiento y cultura, 503504, 113-128

Bindel, Julie (2017). The Pimping of Prostitution. Abolishing the Sew Work Myth. Londres: Palgrave.

Bredy, Erica; Robles Gallego, Ana; Blanca Pérez, Beatriz; Gallardo García, Begoña y Vargas Verde, Lucía (2014). Estudio sobre la trata, la prostitución y otras formas de explotación sexual en la ciudad de Sevilla. Sevilla: Ayuntamiento de Sevilla.

Castellanos, Esther y Ranea, Beatriz (2013). Investigación sobre prostitución y trata con fines de explotación sexual. Madrid: Aprosers, Ministerio de Empleo y Seguridad Social.

Cobo, Rosa (2017). La prostitución en el corazón del capitalismo. Madrid: La Catarata.

Cobo, Rosa (2007). Ponencia en Informe de la ponencia sobre la prostitución en nuestro país. Madrid: Congreso de los Diputados, Gobierno de España. 
De Miguel, Ana (2014). La prostitución de mujeres, una escuela de desigualdad humana. Dilemata: Revista Internacional de Éticas Aplicadas 16, 7-30.

EAPN y Llano Ortiz, Juan Carlos (2018). $7^{\circ}$ Informe anual sobre el estado de la pobreza y la exclusión social en España. Madrid: EAPN.

Fernández Viguera, Blanca y Munárriz Gómara, Julia (2008). Diagnóstico de la exclusión social en Navarra. Prostitución y exclusión social. II Plan de Lucha contra la exclusión social en Navarra. Pamplona: Universidad Pública de Navarra.

García Cuesta, Sara; López Sala, Ana; Corrochano Hernández, Elena y Mena Martínez, Luis (2011). Poblaciones mercancía: tráfico y trata de mujeres en España. Madrid: Ministerio de Sanidad, Política Social e Igualdad.

Gimeno, Beatriz (2012). La prostitución. Aportaciones para un debate abierto. Barcelona: Bellaterra.

Izcara-Palacios, Simón Pedro y Karla Lorena Andrade-Rubio (2016). La deportación de mujeres centroamericanas víctimas de trata. Papeles de Población, 90, 173-196. DOI: http:/ /dx.doi.org /10.22185/24487147.2016.90.038

Jeffreys, Sheila (2009). The Industrial Vagina. The Political Economy of the Global Sex Trade. Nueva York: Roudledge.

Kimmel, Michael S. (2018). Introduction: Toward a Sociology of the Superordinate. En Michael Kimmel y Abby L. Ferber (eds.), Privilege: A Reader (pp. 15-26). Nueva York: Routledge.

Marttila, Anne-Marie (2018). Desiring the "Other": Prostitution Clients on a Transnational Red-Light District in the Border Area of Finland, Estonia and Rusia. Gender, Technology and Development 12 (1), 31-51. DOI: https://doi.org/10.1177/097185240701200104

Palomo Cermeño, Eva (2017). La mercantilización de los cuerpos de las mujeres y la doble moral en el pensamiento feminista del siglo XIX: Josephine Butler y el movimiento abolicionista de la prostitución. En De Miguel, Ana y Nuño, Laura (eds.), Elementos para una teoría crítica del sistema prostitucional. Granada: Comares.

Pérez Freire, Silvia y Casado-Neira, David (2015). Sexo, masculinidades y las víctimas expiatorias. Las mujeres en contexto de prostitución en los medios: la mirada desde las ONG. Revista Internacional de Comunicación y Desarrollo, 2, 25-40. DOI: http: / / dx.doi.org/10.15304/ricd.1.2.2650

Posada Kubissa, Luisa (2015). Las mujeres son cuerpo: reflexiones feministas. Investigaciones Feministas, 6, 108-121. DOI: http:/ /dx.doi.org/10.5209/rev_INFE.2015.v6.51382 
Presentación del monográfico “La prostitución: entre viejos privilegios masculinos y nuevos imaginarios neoliberales"

Ranea, Beatriz (2017). (Re)pensar la prostitución desde el análisis crítico de la masculinidad. En De Miguel, Ana y Nuño, Laura (eds.) Elementos para una teoría crítica del sistema prostitucional. Granada: Comares.

Ranea, Beatriz (2016). Analizando la demanda: relación entre masculinidad hegemónica y prostitución femenina. Investigaciones Feministas 7:2, 313-330. DOI: http:/ /dx.doi.org/10.5209/rev_INFE.2016.v7.n1.50746

Sau, Victoria (2000). Diccionario ideológico feminista. Barcelona: Icaria.

Varela, Nuria (2017). Cansadas. Una reacción feminista frente a la nueva misoginia. Barcelona: Ediciones b. 